\title{
Pacific
}

Journal of

Mathematics

\section{AFFINE GROUP SCHEMES OVER SYMMETRIC MONOIDAL} CATEGORIES

ABHISHEK BANERJEE 


\title{
AFFINE GROUP SCHEMES OVER SYMMETRIC MONOIDAL CATEGORIES
}

\author{
ABHISHEK BANERJEE
}

\begin{abstract}
A well known result of Deligne shows that an affine commutative group scheme of rank $r$ is annihilated by its rank. The purpose of this paper is to extend this result to affine group schemes over symmetric monoidal categories.
\end{abstract}

\section{Introduction}

One of the most important results in the study of group schemes is the following, presented in [Tate and Oort 1970].

Theorem 1.1 (Deligne's lemma). Let $G=\operatorname{Spec}(A)$ be an affine commutative group scheme over a commutative, Noetherian ring $k$. Assume that $A$ is a flat $k$-algebra of rank $r \geq 1$. Then, for any $k$-algebra $B$, all elements in the group $G(B)$ have an order dividing $r$.

The purpose of this paper is to obtain an analogous result for group schemes in the relative algebraic geometry over a symmetric monoidal category. More precisely, we let $(\mathbf{C}, \otimes, 1)$ denote an abelian closed symmetric monoidal category. For instance, $\mathbf{C}$ could be the category of sheaves of abelian groups over a topological space, the category of comodules over a flat Hopf algebroid, the derived category of modules over a commutative ring $k$ as well as chain complexes over all these categories. When $\mathbf{C}=k$-Mod, the category of modules over a commutative ring $k$, the algebraic geometry over $\mathbf{C}$ reduces to the usual algebraic geometry over $\operatorname{Spec}(k)$.

Given $(\mathbf{C}, \otimes, 1)$ as above, we refer to commutative and unital monoids in $\mathbf{C}$ as algebras in $\mathbf{C}$. Then, we define an affine commutative group scheme $G$ free of finite rank over $\mathbf{C}$ to be a covariant functor from algebras in $\mathbf{C}$ to the category of abelian groups that satisfies certain conditions (see Definition 3.2 and Definition 3.3). The main result of this article is the following theorem:

The author is happy to acknowledge support from the Max-Planck-Institut für Mathematik, Bonn, where most of this paper was written.

MSC2010: 14L15, 18D10.

Keywords: group schemes, symmetric monoidal categories. 
Theorem 1.2. Let $(\mathbf{C}, \otimes, 1)$ be an abelian, closed, $\mathbb{C}$-linear symmetric monoidal category and let $G$ be an affine commutative group scheme over $\mathbf{C}$ free and of finite rank $r \geq 1$. Then, for any algebra $B$ in $\mathbf{C}$ and any element $u$ in the group $G(B)$, we have $u^{r}=1_{B}$, where $1_{B}$ denotes the identity element of $G(B)$. (For the definition of $G(B)$, see (3-5).)

The relative algebraic geometry over a symmetric monoidal category has been developed in various works, such as [Deligne 1990; Hakim 1972; Toën and Vaquié 2009]. It is therefore natural to ask whether arithmetic geometry can be similarly developed in the general framework of symmetric monoidal categories. In particular, since the theory of finite flat group schemes is closely linked to arithmetic (see [Tate 1997], for instance), they are a natural starting point for such a theory. For more on group schemes, we refer the reader to [Demazure and Gabriel 1970].

\section{Notations}

In this section, we introduce notation that we will maintain throughout this paper. We let $(\mathbf{C}, \otimes, 1)$ denote an abelian symmetric monoidal category. Further, we suppose that $\mathbf{C}$ is closed, i.e., for any two objects $X, Y \in \mathbf{C}$, there exists an internal Hom object $\underline{\operatorname{Hom}}(X, Y)$ in $\mathbf{C}$ such that the functor

$$
Z \mapsto \operatorname{Hom}(Z \otimes X, Y)
$$

from $\mathbf{C}$ to the category of sets is represented by $\underline{\operatorname{Hom}}(X, Y)$. Here, we also note that, for any objects $X, Y, Z$ and $W$ in $\mathbf{C}$, we have

$$
\begin{aligned}
\operatorname{Hom}(W, \underline{\operatorname{Hom}}(Z, \underline{\operatorname{Hom}}(X, Y))) & \cong \operatorname{Hom}(W \otimes Z, \underline{\operatorname{Hom}}(X, Y)) \\
& \cong \operatorname{Hom}(W \otimes Z \otimes X, Y) \\
& \cong \operatorname{Hom}(W, \underline{\operatorname{Hom}}(Z \otimes X, Y)) .
\end{aligned}
$$

Hence, it follows from Yoneda's lemma that we have a natural isomorphism

$$
\underline{\operatorname{Hom}}(Z, \underline{\operatorname{Hom}}(X, Y)) \cong \underline{\operatorname{Hom}}(Z \otimes X, Y)
$$

for any $X, Y, Z$ and $W$ in $\mathbf{C}$. Further, since $\mathbf{C}$ is an abelian category, $\mathbf{C}$ is additive and hence finite direct sums coincide with finite direct products in $\mathbf{C}$. For any object $X \in \mathbf{C}$ and any integer $r \in \mathbb{Z}, r>0$, we let $X^{r}$ denote the direct sum (or direct product) of $r$-copies of $X$ in $\mathbf{C}$.

By an algebra in $\mathbf{C}$, we will always mean a commutative monoid object with unit in $\mathbf{C}$. The category of algebras in $\mathbf{C}$ will be denoted by Alg. More precisely, an algebra in $\mathbf{C}$ is an object $A$ in $\mathbf{C}$ with a multiplication map $m_{A}: A \otimes A \rightarrow A$ and a unit map $u_{A}: 1 \rightarrow A$. satisfying the compatibility conditions for making $A$ a commutative monoid with unit (see [Mac Lane 1998], for instance). 
For any algebra $A$, we let $A$-Mod denote the category of $A$-modules in $\mathbf{C}$. Then, each $\left(A-\operatorname{Mod}, \otimes_{A}, A\right)$ is also a closed symmetric monoidal category. Given any $A$-modules $M$ and $N$, we will denote by $\operatorname{Hom}_{A}(M, N)$ the set of morphisms from $M$ to $N$ in $A$-Mod and the internal Hom object by $\underline{\operatorname{Hom}}_{A}(M, N)$. It is clear that $\operatorname{Hom}_{A}(M, N)$ is an abelian group. Further, the category of unitary commutative monoids in $A$-Mod will be denoted by $A$-Alg. For any two $A$-algebras $B$ and $B^{\prime}$, we will denote by $\operatorname{Hom}_{A-A l g}\left(B, B^{\prime}\right)$ the set of $A$-algebra morphisms from $B$ to $B^{\prime}$. If $f: A \rightarrow B$ is a morphism of algebras, for any $A$-module $M$ and $B$-module $N$, we have natural isomorphisms

$$
T: \operatorname{Hom}_{A}(M, N) \cong \operatorname{Hom}_{B}\left(M \otimes_{A} B, N\right)
$$

described as follows: given $g \in \operatorname{Hom}_{A}(M, N)$, we define

$$
T(g) \in \operatorname{Hom}_{B}\left(M \otimes_{A} B, N\right)
$$

as the composition

$$
T(g): M \otimes_{A} B \stackrel{g \otimes_{A} 1}{\longrightarrow} N \otimes_{A} B \longrightarrow N,
$$

where the morphism $N \otimes_{A} B \rightarrow N$ in (2-5) follows from the $B$-module structure of $N$. Conversely, given $h \in \operatorname{Hom}_{B}\left(M \otimes_{A} B, N\right)$, it is clear that we have $h=T\left(h^{\prime}\right)$, where $h^{\prime} \in \operatorname{Hom}_{A}(M, N)$ is defined as the composition

$$
h^{\prime}: M \cong M \otimes_{A} A \stackrel{1 \otimes_{A} f}{\longrightarrow} M \otimes_{A} B \stackrel{h}{\longrightarrow} N .
$$

Furthermore, for any object $X$ in $A-M o d$, we note that

$$
\begin{aligned}
\operatorname{Hom}_{A}\left(X, \underline{\operatorname{Hom}}_{A}(M, N)\right) & \cong \operatorname{Hom}_{A}\left(X \otimes_{A} M, N\right) \\
& \cong \operatorname{Hom}_{B}\left(X \otimes_{A} M \otimes_{A} B, N\right) \\
& \cong \operatorname{Hom}_{B}\left(\left(X \otimes_{A} B\right) \otimes_{B}\left(M \otimes_{A} B\right), N\right) \\
& \cong \operatorname{Hom}_{B}\left(X \otimes_{A} B, \underline{\operatorname{Hom}}_{B}\left(M \otimes_{A} B, N\right)\right) \\
& \cong \operatorname{Hom}_{A}\left(X, \underline{\operatorname{Hom}}_{B}\left(M \otimes_{A} B, N\right)\right) .
\end{aligned}
$$

Using (2-7), it follows from Yoneda's lemma that we have natural isomorphisms in A-Mod:

$$
\underline{\operatorname{Hom}}_{A}(M, N) \cong \underline{\operatorname{Hom}}_{B}\left(M \otimes_{A} B, N\right) .
$$

\section{Affine group schemes}

Let $(\mathbf{C}, \otimes, 1)$ be an abelian, closed, symmetric monoidal category as described in Section 2 and let $A$ be an algebra in $\mathbf{C}$. Then, it is well known (see, for instance, [May 2001]) that the collection of endomorphisms $\operatorname{Hom}_{A}(A, A)$ is an ordinary commutative ring with identity. We start with the following result. 
Proposition 3.1. Let $A$ be an algebra in $\mathbf{C}$. Then, there is a natural isomorphism

$$
\operatorname{Hom}(1, A) \stackrel{\sim}{\longrightarrow} \operatorname{Hom}_{A}(A, A) .
$$

Proof. Define a map $S: \operatorname{Hom}(1, A) \rightarrow \operatorname{Hom}_{A}(A, A)$ thus: given $f \in \operatorname{Hom}(1, A)$, let $S(f) \in \operatorname{Hom}_{A}(A, A)$ be the composition

$$
A \stackrel{\sim}{\longrightarrow} A \otimes 1 \stackrel{1 \otimes f}{\longrightarrow} A \otimes A \stackrel{m_{A}}{\longrightarrow} A,
$$

where $m_{A}: A \otimes A \rightarrow A$ in (3-2) is the multiplication map on the algebra $A$. Conversely, we define a map $T: \operatorname{Hom}_{A}(A, A) \rightarrow \operatorname{Hom}(1, A)$ as follows: given $g \in \operatorname{Hom}_{A}(A, A)$, we let $T(g) \in \operatorname{Hom}(1, A)$ denote the composition

$$
1 \stackrel{u_{A}}{\longrightarrow} A \stackrel{g}{\longrightarrow} A
$$

where the map $u_{A}: 1 \rightarrow A$ in (3-3) is the "unit map" for the algebra $A$. It is easy to check that the associations $S$ and $T$ are inverse to each other and hence we have an isomorphism $\operatorname{Hom}(1, A) \stackrel{\sim}{\longrightarrow} \operatorname{Hom}_{A}(A, A)$.

Following [Toën and Vaquié 2009], we define $\operatorname{Aff}_{\mathbf{C}}:=\operatorname{Alg}^{o p}$ to be the category of affine schemes over $\mathbf{C}$. For any algebra $A$ in $\mathbf{C}$, we let $\operatorname{Spec}(A)$ denote the corresponding object of $\operatorname{Aff}_{\mathbf{C}}$. Further, we denote by $\mathfrak{s p e c}(A)$ the (contravariant) functor on $\operatorname{Aff}_{\mathbf{C}}$ represented by $\operatorname{Spec}(A)$.

Definition 3.2. Let $(\mathbf{C}, \otimes, 1)$ be as above and let Set denote the category of sets. An affine group scheme over $\mathbf{C}$ is a representable functor

$$
G=\mathfrak{s p e c}(A): \operatorname{Aff}_{\mathbf{C}} \rightarrow \text { Set },
$$

equipped with a composition map $m_{G}: G \times G \rightarrow G$, an inverse map $i_{G}: G \rightarrow G$ and a unit map $e_{G}: \mathfrak{s p e c}(1) \rightarrow G$ of functors satisfying the group axioms (see [Waterhouse 1979, § 1.4], for instance).

From Yoneda's lemma it follows that if $G=\mathfrak{s p e c}(A)$ is an affine group scheme in the sense of Definition 3.2, then $A$ is an algebra in $\mathbf{C}$ equipped with a comultiplication $\Delta_{A}: A \rightarrow A \otimes A$, an antipode $i_{A}: A \rightarrow A$ and a counit $\epsilon_{A}: A \rightarrow 1$ that gives $A$ the structure of a Hopf algebra in $\mathbf{C}$. Further, if Grp denotes the category of groups, we can also express $G$ as a functor from algebras in $\mathbf{C}$ to groups:

$$
G: \operatorname{Alg} \rightarrow \operatorname{Grp}, \quad G(B):=\operatorname{Hom}_{\mathrm{Aff}_{\mathrm{C}}}(\operatorname{Spec}(B), \operatorname{Spec}(A))=\operatorname{Hom}_{\mathrm{Alg}}(A, B) .
$$

Further, since the comultiplication $\Delta_{A}: A \rightarrow A \otimes A$ in Alg corresponds to the composition $m_{G}: G \times G \rightarrow G$, it follows that $A$ is cocommutative if and only if, for all algebras $B$ in $\mathbf{C}$, the group $G(B)$ is abelian. In this case, we will say that $G=\mathfrak{s p e c}(A)$ is an affine commutative group scheme over $\mathbf{C}$. 
Definition 3.3. Let $G=\mathfrak{s p e c}(A)$ be an affine commutative group scheme over $\mathbf{C}$. Then, we say that $G$ is free of finite rank $r \in \mathbb{Z}, r>0$ if $A \cong 1^{r}$ as objects of $\mathbf{C}$, where $1^{r}$ denotes the direct sum of $r$-copies of the unit object 1 of $\mathbf{C}$.

Further, suppose that $B$ is an algebra in $\mathbf{C}$ and let $B^{\prime}$ be a $B$-algebra. Then, $B^{\prime}$ is said to be a locally free $B$-algebra of rank $r$ if $B^{\prime} \cong B^{r}$ as $B$-modules. In case $B=1$, we will simply say that $B^{\prime}$ is a locally free algebra of rank $r$.

From now onwards we will always let $G=\mathfrak{s p e c}(A)$ be an affine commutative group scheme over $\mathbf{C}$ that is free of finite rank $r$. We also define $A^{\prime}:=\underline{\operatorname{Hom}}(A, 1)$. Then, it is clear that for any object $X$ in $\mathbf{C}$, we have natural isomorphisms (3-6) $\underline{\operatorname{Hom}}(A, X) \cong \underline{\operatorname{Hom}}\left(1^{r}, X\right) \cong \bigoplus^{r} \underline{\operatorname{Hom}}(1, X) \cong \underline{\operatorname{Hom}}(A, 1) \otimes X \cong A^{\prime} \otimes X$.

Proposition 3.4. Let $G=\mathfrak{s p e c}(A)$ be an affine commutative group scheme over $\mathrm{C}$ that is free of finite rank $r$. Then, $A^{\prime}:=\underline{\operatorname{Hom}}(A, 1)$ is a commutative and cocommutative Hopf algebra in $\mathbf{C}$ and is also a locally free algebra of rank $r$.

Proof. Since $G=\mathfrak{s p e c}(A)$ is an affine commutative group scheme, we know that $A$ is a commutative and cocommutative Hopf algebra in C. From (2-3) and (3-6), it follows that

$$
A^{\prime} \otimes A^{\prime} \cong \underline{\operatorname{Hom}}(A, \underline{\operatorname{Hom}}(A, 1)) \cong \underline{\operatorname{Hom}}(A \otimes A, 1) .
$$

It is clear that the multiplication $m_{A}: A \otimes A \rightarrow A$ induces a map

$$
A^{\prime}=\underline{\operatorname{Hom}}(A, 1) \rightarrow \underline{\operatorname{Hom}}(A \otimes A, 1),
$$

while the comultiplication $\Delta_{A}: A \rightarrow A \otimes A$ induces

$$
\underline{\operatorname{Hom}}(A \otimes A, 1) \rightarrow \underline{\operatorname{Hom}}(A, 1)=A^{\prime} .
$$

Combining this with (3-7), we obtain a natural multiplication $m_{A^{\prime}}: A^{\prime} \otimes A^{\prime} \rightarrow A^{\prime}$ and a natural comultiplication $\Delta_{A^{\prime}}: A^{\prime} \rightarrow A^{\prime} \otimes A^{\prime}$ on $A^{\prime}$. The unit $u_{A^{\prime}}: 1 \rightarrow A^{\prime}$, the counit $\epsilon_{A^{\prime}}: A^{\prime} \rightarrow 1$ and the antipode $i_{A^{\prime}}: A^{\prime} \rightarrow A^{\prime}$ on $A^{\prime}$ are obtained by dualizing $\epsilon_{A}: A \rightarrow 1, u_{A}: 1 \rightarrow A$ and $i_{A}: A \rightarrow A$ respectively. It is clear that these maps make $A^{\prime}$ into a commutative and cocommutative Hopf algebra.

Finally, since $A \cong 1^{r}$, it follows that $A^{\prime} \cong \underline{\operatorname{Hom}}(A, 1) \cong 1^{r}$ and hence $A^{\prime}$ is also a locally free algebra of rank $r$.

Proposition 3.5. Let $G=\mathfrak{s p e c}(A)$ be an affine commutative group scheme over $\mathbf{C}$ that is free of finite rank $r$. Let $A^{\prime}=\underline{\operatorname{Hom}}(A, 1)$. Then:

(a) There are natural isomorphisms

$$
\operatorname{Hom}(A, 1) \cong \operatorname{Hom}\left(1, A^{\prime}\right) \cong \operatorname{Hom}_{A^{\prime}}\left(A^{\prime}, A^{\prime}\right) .
$$

Further, each of the objects in (3-8) carries a comultiplication structure that is compatible with the isomorphisms in (3-8). 
(b) There are natural isomorphisms

$$
\operatorname{Hom}(A, A) \cong \operatorname{Hom}\left(1, A^{\prime} \otimes A\right) \cong \operatorname{Hom}_{A^{\prime} \otimes A}\left(A^{\prime} \otimes A, A^{\prime} \otimes A\right) .
$$

Further, each of the objects in (3-9) carries a comultiplication structure that is compatible with the isomorphisms in (3-9).

Proof. (a) Since $A^{\prime}=\underline{\operatorname{Hom}}(A, 1)$, it is clear that $\operatorname{Hom}(A, 1) \cong \operatorname{Hom}\left(1, A^{\prime}\right)$. Since Proposition 3.4 shows that $A^{\prime}$ is also an algebra, the isomorphism $\operatorname{Hom}\left(1, A^{\prime}\right) \cong$ $\operatorname{Hom}_{A^{\prime}}\left(A^{\prime}, A^{\prime}\right)$ follows from Proposition 3.1.

We now describe the comultiplication structure on $\operatorname{Hom}_{A^{\prime}}\left(A^{\prime}, A^{\prime}\right)$. Given $f$ in $\operatorname{Hom}_{A^{\prime}}\left(A^{\prime}, A^{\prime}\right)$, we can define a morphism

$$
\delta_{1}(f) \in \operatorname{Hom}_{A^{\prime} \otimes A^{\prime}}\left(A^{\prime} \otimes A^{\prime}, A^{\prime} \otimes A^{\prime}\right)
$$

as follows:

$(3-10) \quad \delta_{1}(f): A^{\prime} \otimes A^{\prime} \stackrel{\sim}{\longrightarrow} 1 \otimes A^{\prime} \otimes A^{\prime} \longrightarrow A^{\prime} \otimes A^{\prime} \otimes A^{\prime} \stackrel{f \otimes 1 \otimes 1}{\longrightarrow} A^{\prime} \otimes A^{\prime} \otimes A^{\prime}$

$$
\stackrel{\Delta_{A^{\prime}} \otimes 1 \otimes 1}{\longrightarrow} A^{\prime} \otimes A^{\prime} \otimes A^{\prime} \otimes A^{\prime} \stackrel{m_{13}^{\prime} \otimes m_{24}^{\prime}}{\longrightarrow} A^{\prime} \otimes A^{\prime},
$$

where $m_{i j}^{\prime}: A^{\prime} \otimes A^{\prime} \rightarrow A^{\prime}$ in (3-10) denotes the multiplication $m_{A^{\prime}}: A^{\prime} \otimes A^{\prime} \rightarrow A^{\prime}$ on $A^{\prime}$ applied to the $i$-th and $j$-th copy of $A^{\prime}$ appearing in the term $A^{\prime} \otimes A^{\prime} \otimes A^{\prime} \otimes A^{\prime}$ in (3-10). Since $A^{\prime}$ is a locally free algebra of rank $r$, we have natural isomorphisms

$$
\operatorname{Hom}_{A^{\prime}}\left(A^{\prime}, A^{\prime}\right) \otimes \operatorname{Hom}_{A^{\prime}}\left(A^{\prime}, A^{\prime}\right) \cong \operatorname{Hom}_{A^{\prime} \otimes A^{\prime}}\left(A^{\prime} \otimes A^{\prime}, A^{\prime} \otimes A^{\prime}\right) .
$$

Using (3-10) and (3-11), we have a comultiplication

$$
\delta_{1}: \operatorname{Hom}_{A^{\prime}}\left(A^{\prime}, A^{\prime}\right) \rightarrow \operatorname{Hom}_{A^{\prime}}\left(A^{\prime}, A^{\prime}\right) \otimes \operatorname{Hom}_{A^{\prime}}\left(A^{\prime}, A^{\prime}\right) .
$$

Considering the comultiplication $\Delta_{A^{\prime}}: A^{\prime} \rightarrow A^{\prime} \otimes A^{\prime}$ on $A^{\prime}$, we have an induced map

(3-13) $\delta_{2}: \operatorname{Hom}\left(1, A^{\prime}\right) \stackrel{\operatorname{Hom}\left(1, \Delta_{A^{\prime}}\right)}{\longrightarrow} \operatorname{Hom}\left(1, A^{\prime} \otimes A^{\prime}\right) \cong \operatorname{Hom}\left(1, A^{\prime}\right) \otimes \operatorname{Hom}\left(1, A^{\prime}\right)$,

where the last isomorphism follows from the fact that $A^{\prime}$ is a locally free algebra. From (3-10), (3-13), and the construction of the isomorphism

$$
\operatorname{Hom}\left(1, A^{\prime}\right) \cong \operatorname{Hom}_{A^{\prime}}\left(A^{\prime}, A^{\prime}\right)
$$

in Proposition 3.1 applied to $A^{\prime}$, it follows that the comultiplications $\delta_{1}$ and $\delta_{2}$ are compatible with the isomorphism $\operatorname{Hom}\left(1, A^{\prime}\right) \cong \operatorname{Hom}_{A^{\prime}}\left(A^{\prime}, A^{\prime}\right)$. Finally, since the comultiplication

$$
\delta_{3}: \operatorname{Hom}(A, 1) \rightarrow \operatorname{Hom}(A \otimes A, 1) \cong \operatorname{Hom}(A, 1) \otimes \operatorname{Hom}(A, 1)
$$


is induced by the multiplication $m_{A}: A \otimes A \rightarrow A$ on $A$ and $m_{A}$ induces the comultiplication $\Delta_{A^{\prime}}: A^{\prime} \rightarrow A^{\prime} \otimes A^{\prime}$ on $A^{\prime}$, the maps $\delta_{2}$ and $\delta_{3}$ are compatible with the isomorphism $\operatorname{Hom}(A, 1) \cong \operatorname{Hom}\left(1, A^{\prime}\right)$.

(b) From (2-4), it follows that

$$
\begin{gathered}
\operatorname{Hom}\left(1, A^{\prime} \otimes A\right) \cong \operatorname{Hom}_{A}\left(A, A^{\prime} \otimes A\right) \cong \operatorname{Hom}_{A^{\prime} \otimes A}\left(A^{\prime} \otimes A, A^{\prime} \otimes A\right), \\
\operatorname{Hom}(A, A) \cong \operatorname{Hom}_{A}(A \otimes A, A) .
\end{gathered}
$$

We also note that, using (2-8) and (3-6), we have

$$
\underline{\operatorname{Hom}}_{A}(A \otimes A, A) \cong \underline{\operatorname{Hom}}(A, A) \cong A^{\prime} \otimes A
$$

From (3-16), it follows that the dual of $A \otimes A$ in the category $A$-Mod is $A^{\prime} \otimes A$. Further, the comultiplication $\Delta_{A}: A \rightarrow A \otimes A$ induces a comultiplication

$$
\Delta_{A}^{A}: A \otimes A \rightarrow(A \otimes A) \otimes_{A}(A \otimes A)
$$

on the $A$-algebra $A \otimes A$ as follows:

$$
\Delta_{A}^{A}:=\Delta_{A} \otimes 1_{A}: A \otimes A \rightarrow A \otimes A \otimes A \cong(A \otimes A) \otimes_{A}(A \otimes A)
$$

making $A \otimes A$ into a Hopf algebra in $A$-Mod. Applying the result of part (a) to the object $A \otimes A$ in $A-M o d$, we have compatible comultiplications on each of the following isomorphic objects

(3-18) $\operatorname{Hom}_{A}(A \otimes A, A) \cong \operatorname{Hom}_{A}\left(A, A^{\prime} \otimes A\right) \cong \operatorname{Hom}_{A^{\prime} \otimes A}\left(A^{\prime} \otimes A, A^{\prime} \otimes A\right)$.

Using the isomorphisms in (3-15), we have compatible induced comultiplications on each of the following isomorphic objects:

$\delta_{1}^{A}: \operatorname{Hom}_{A^{\prime} \otimes A}\left(A^{\prime} \otimes A, A^{\prime} \otimes A\right)$

$\rightarrow \operatorname{Hom}_{A^{\prime} \otimes A}\left(A^{\prime} \otimes A, A^{\prime} \otimes A\right) \otimes \operatorname{Hom}_{A^{\prime} \otimes A}\left(A^{\prime} \otimes A, A^{\prime} \otimes A\right)$, $\delta_{2}^{A}: \operatorname{Hom}\left(1, A^{\prime} \otimes A\right) \rightarrow \operatorname{Hom}\left(1, A^{\prime} \otimes A\right) \otimes \operatorname{Hom}\left(1, A^{\prime} \otimes A\right)$,

$\delta_{3}^{A}: \operatorname{Hom}(A, A) \rightarrow \operatorname{Hom}(A, A) \otimes \operatorname{Hom}(A, A)$.

\section{Norm map and grouplike elements}

From now onwards, we will assume that the closed abelian symmetric monoidal category $(\mathbf{C}, \otimes, 1)$ is $\mathbb{C}$-linear. As before, we let $G=\mathfrak{s p e c}(A)$ be an affine commutative group scheme that is free of finite rank $r$. Let $A^{\prime}=\underline{\operatorname{Hom}}(A, 1)$. Given any algebra $B$ in $\mathbf{C}$, we will construct a map

$$
N_{B}: \operatorname{Hom}_{B \otimes A}(B \otimes A, B \otimes A) \rightarrow \operatorname{Hom}_{B}(B, B),
$$

which corresponds to the norm map in the context of ordinary $\mathbb{Z}$-algebras. We will refer to the $B$-algebra $B \otimes A$ as $B_{A}$. 
Let $M$ be a $B$-module. Since the category $B$-Mod is $\mathbb{C}$-linear, the notion of exterior product extends to it. For any integer $n \geq 1$, we can consider the tensor product $M^{\otimes_{B} n}:=M \otimes_{B} M \otimes_{B} \otimes_{B} \cdots \otimes_{B} M$ (n-times). Then, the symmetric group $S_{n}$ acts on $M^{\otimes_{B} n}$ by permutations, i.e., for each $\sigma \in S_{n}$, we have an induced map $\sigma: M^{\otimes_{B} n} \rightarrow M^{\otimes_{B} n}$ of $B$-modules. We then consider the morphism

$$
q_{M}^{n}: M^{\otimes_{B} n} \rightarrow M^{\otimes_{B} n} \quad q_{M}^{n}:=1-\frac{1}{n !} \sum_{\sigma \in S_{n}} \operatorname{sgn}(\sigma) \sigma .
$$

It is clear that the morphism $q_{M}^{n} \in \operatorname{Hom}_{B}\left(M^{\otimes_{B} n}, M^{\otimes_{B} n}\right)$ is an idempotent. Since $\mathbf{C}$ is an abelian category, we can form the cokernel of $q_{M}^{n}$, which we denote by $\bigwedge_{B}^{n} M$. Further, since $q_{M}^{n}$ is an idempotent, the cokernel $\bigwedge_{B}^{n} M$ is a direct summand of $M^{\otimes_{B} n}$. It follows that for any $n \geq 1, q_{M}^{n}$ induces a morphism

$$
\operatorname{Hom}\left(\bigwedge_{B}^{n}\right)(M): \operatorname{Hom}_{B}\left(M^{\otimes_{B} n}, M^{\otimes_{B} n}\right) \rightarrow \operatorname{Hom}_{B}\left(\bigwedge_{B}^{n} M, \bigwedge_{B}^{n} M\right) .
$$

In particular, therefore, taking $M=B_{A}$ and $n=r$, we have a map

$$
\operatorname{Hom}\left(\bigwedge_{B}^{r}\right)\left(B_{A}\right): \operatorname{Hom}_{B}\left(B_{A}^{\otimes_{B} r}, B_{A}^{\otimes_{B} r}\right) \rightarrow \operatorname{Hom}_{B}\left(\bigwedge_{B}^{r} B_{A}, \bigwedge_{B}^{r} B_{A}\right) .
$$

Also, for any objects $X, Y$ in $B-M o d$, the exterior product satisfies

$$
\bigwedge_{B}^{n}(X \oplus Y) \cong \bigoplus_{k+l=n} \wedge_{B}^{k} X \otimes_{B} \bigwedge_{B}^{l} Y .
$$

In the situation above, since $A$ is a locally free algebra of rank $r$, i.e., $A \cong 1^{r}$, it follows that $B_{A}=B \otimes A \cong B^{r}$. Hence, $B_{A}$ is a locally free $B$-algebra of rank $r$.

Lemma 4.1. Let $G=\mathfrak{s p e c}(A)$ be an affine commutative group scheme free of finite rank $r$. Let $B$ be an algebra in $\mathbf{C}$. Then, there exists a natural isomorphism $\wedge_{B}^{r} B_{A} \cong B$ of $B$-modules.

Proof. For any $k \geq 2$, we consider the morphism

$$
q_{B}^{k}: B^{\otimes_{B} k} \rightarrow B^{\otimes_{B} k} .
$$

Since $B$ is a commutative monoid, any morphism $\sigma: B^{\otimes_{B} k} \cong B \rightarrow B^{\otimes_{B} k} \cong B$ induced by some $\sigma \in S_{k}$ corresponds to the identity map $1_{B}: B \rightarrow B$. Since $\sum_{\sigma \in S_{k}} \operatorname{sgn}(\sigma)=0$, it follows that $q_{B}^{k}$ is the identity. Hence

$$
\bigwedge_{B}^{k} B:=\operatorname{Coker}\left(q_{B}^{k}\right)=0 .
$$

It follows from (4-5) and (4-7) that

$$
\begin{aligned}
\bigwedge_{B}^{r} B_{A} & \cong \bigwedge_{B}^{r} B^{r} \\
& \cong \bigoplus_{k_{1}+k_{2}+\cdots+k_{r}=r} \bigwedge_{B}^{k_{1}} B \otimes_{B} \cdots \otimes_{B} \bigwedge_{B}^{k_{r}} B \cong B \otimes_{B} \cdots \otimes_{B} B \cong B
\end{aligned}
$$


Proposition 4.2. Let $G=\mathfrak{s p e c}(A)$ be an affine commutative group scheme free of finite rank $r$. Let $B$ be an algebra in $\mathbf{C}$. Then, there exists a norm map

$$
N_{B}: \operatorname{Hom}_{B \otimes A}(B \otimes A, B \otimes A) \rightarrow \operatorname{Hom}_{B}(B, B)
$$

that is compatible with composition on $\operatorname{Hom}_{B \otimes A}(B \otimes A, B \otimes A)$ and $\operatorname{Hom}_{B}(B, B)$. Proof. We set $B_{A}=B \otimes A$ as above. First, we note that we have a forgetful map (4-10) $\operatorname{Hom}_{B \otimes A}(B \otimes A, B \otimes A) \rightarrow \operatorname{Hom}_{B}(B \otimes A, B \otimes A)=\operatorname{Hom}_{B}\left(B_{A}, B_{A}\right)$. Following this, we consider the morphism

$$
\operatorname{Hom}_{B}\left(B_{A}, B_{A}\right) \rightarrow \operatorname{Hom}_{B}\left(B_{A}^{\otimes r}, B_{A}^{\otimes r}\right), \quad f \mapsto f^{\otimes r} .
$$

From (4-4) and Lemma 4.1, we have

(4-12) $\operatorname{Hom}\left(\bigwedge_{B}^{r}\right)\left(B_{A}\right): \operatorname{Hom}_{B}\left(B_{A}^{\otimes r}, B_{A}^{\otimes r}\right) \rightarrow \operatorname{Hom}_{B}\left(\bigwedge_{B}^{r} B_{A}, \bigwedge_{B}^{r} B_{A}\right)$

$$
\cong \operatorname{Hom}_{B}(B, B) \text {. }
$$

Composing the morphisms in (4-10), (4-11) and (4-12), we have the map

$$
N_{B}: \operatorname{Hom}_{B \otimes A}(B \otimes A, B \otimes A) \rightarrow \operatorname{Hom}_{B}(B, B) .
$$

Finally, it is clear from the construction that $N_{B}$ is compatible with composition on $\operatorname{Hom}_{B \otimes A}(B \otimes A, B \otimes A)$ and $\operatorname{Hom}_{B}(B, B)$.

By composing the maps in (4-11) and (4-12) in the proof of Proposition 4.2, it follows that we have a norm map $\operatorname{Hom}_{B}(B \otimes A, B \otimes A) \rightarrow \operatorname{Hom}_{B}(B, B)$ for any algebra $B$ in $\mathbf{C}$ which we will continue to denote by $N_{B}$.

Let $f: B \rightarrow C$ be a morphism of algebras in $\mathbf{C}$. Then, it follows from base change that $f$ induces maps

$$
\operatorname{Hom}(f): \operatorname{Hom}_{B}(B, B) \rightarrow \operatorname{Hom}_{C}(C, C),
$$

$$
\operatorname{Hom}_{A}(f \otimes 1): \operatorname{Hom}_{B \otimes A}(B \otimes A, B \otimes A) \rightarrow \operatorname{Hom}_{C \otimes A}(C \otimes A, C \otimes A) .
$$

Further, since the morphisms (4-10), (4-11) and (4-12) are all natural with respect to base change, the following diagram is commutative:

$$
\begin{array}{ccc}
\operatorname{Hom}_{B \otimes A}(B \otimes A, B \otimes A) & \stackrel{\operatorname{Hom}_{A}(f \otimes 1)}{\longrightarrow} & \operatorname{Hom}_{C \otimes A}(C \otimes A, C \otimes A) \\
N_{B} \downarrow & & N_{C} \downarrow \\
\operatorname{Hom}_{B}(B, B) & \stackrel{\operatorname{Hom}(f)}{\longrightarrow} & \operatorname{Hom}_{C}(C, C)
\end{array}
$$

Lemma 4.3. Let $G=\mathfrak{s p e c}(A)$ be an affine commutative group scheme free of finite rank $r$. Then, for any algebra $B$ in $\mathbf{C}$, we have natural isomorphisms

$$
\operatorname{Hom}_{\mathrm{Alg}}(A, B) \cong \operatorname{Hom}_{B-A l g}(A \otimes B, B) .
$$


Proof. We know that we have an isomorphism

$$
T: \operatorname{Hom}(A, B) \stackrel{\cong}{\longrightarrow} \operatorname{Hom}_{B}(A \otimes B, B) .
$$

Suppose that $f: A \rightarrow B$ is a morphism of algebras. Then, $f \otimes 1: A \otimes B \rightarrow B \otimes B$ is a morphism of $B$-algebras. Further, the multiplication $m_{B}: B \otimes B \rightarrow B$ is also a map of $B$-algebras. It follows that

$$
T(f)=m_{B} \circ(f \otimes 1): A \otimes B \stackrel{f \otimes 1}{\longrightarrow} B \otimes B \stackrel{m_{B}}{\longrightarrow} B
$$

is a morphism of $B$-algebras. Hence, $T$ restricts to a morphism

$$
T^{\text {alg }}: \operatorname{Hom}_{\mathrm{Alg}}(A, B) \rightarrow \operatorname{Hom}_{B-A l g}(A \otimes B, B) .
$$

Next, we choose some $g \in \operatorname{Hom}_{B-A l g}(A \otimes B, B) \subseteq \operatorname{Hom}_{B}(A \otimes B, B)$. Then, it follows that $g=T(f)$, where $f$ is given by the composition

$$
f: A \cong A \otimes 1 \stackrel{1 \otimes e_{B}}{\longrightarrow} A \otimes B \stackrel{g}{\longrightarrow} B .
$$

Here $e_{B}: 1 \rightarrow B$ is the unit map of the algebra $B$. Since both maps in (4-19) are morphisms of algebras, $f \in \operatorname{Hom}(A, B)$ is actually a morphism of algebras. It follows that $T^{\text {alg }}$ is a surjection. Further, since $T^{\text {alg }}$ is obtained by restricting the isomorphism $T, T^{\text {alg }}$ must be injective. Hence, we have an isomorphism

$$
T^{\text {alg }}: \operatorname{Hom}_{\mathrm{Alg}}(A, B) \stackrel{\cong}{\longrightarrow} \operatorname{Hom}_{B-A l g}(A \otimes B, B) .
$$

Proposition 4.4. Let $G=\mathfrak{s p e c}(A)$ be an affine commutative group scheme free of finite rank $r$. Let $A^{\prime}=\underline{\operatorname{Hom}}(A, 1)$. Then:

(a) Let $g \in \operatorname{Hom}(A, 1)$ be a morphism that corresponds to $h \in \operatorname{Hom}_{A^{\prime}}\left(A^{\prime}, A^{\prime}\right)$ under the isomorphism $\operatorname{Hom}_{A^{\prime}}\left(A^{\prime}, A^{\prime}\right) \cong \operatorname{Hom}(A, 1)$ in (3-8). Then, $g: A \rightarrow 1$ is a morphism of algebras if and only if $\delta_{1}(h)=h \otimes h$ in the notation of Proposition 3.5.

(b) Let $g \in \operatorname{Hom}(A, A)$ be a morphism that corresponds to

$$
h \in \operatorname{Hom}_{A^{\prime} \otimes A}\left(A^{\prime} \otimes A, A^{\prime} \otimes A\right)
$$

under the isomorphism $\operatorname{Hom}_{A^{\prime} \otimes A}\left(A^{\prime} \otimes A, A^{\prime} \otimes A\right) \cong \operatorname{Hom}(A, A)$ in (3-9). Then, $g: A \rightarrow A$ is a morphism of algebras if and only if $\delta_{1}^{A}(h)=h \otimes h$ in the notation of Proposition 3.5.

Proof. We maintain the notation of the proof of Proposition 3.5.

(a) Using Proposition 3.5(a), it suffices to check that $g \in \operatorname{Hom}(A, 1)$ is a morphism of algebras if and only if $\delta_{3}(g)=g \otimes g$ where $\delta_{3}$ denotes the comultiplication on $\operatorname{Hom}(A, 1)$. 
By definition of $\delta_{3}$ in (3-14), we know that $\delta_{3}(g)$ is equal to the composition

$$
A \otimes A \stackrel{m_{A}}{\longrightarrow} A \stackrel{g}{\longrightarrow} 1 \stackrel{\cong}{\longrightarrow} 1 \otimes 1 .
$$

It is immediate from (4-21) that $\delta_{3}(g)=g \otimes g$ if and only if $g: A \rightarrow 1$ is a morphism of algebras.

(b) Using Proposition 3.5(b), we know that the comultiplication $\delta_{3}^{A}$ on $\operatorname{Hom}(A, A)$ corresponds to the comultiplication $\delta_{1}^{A}$ on $\operatorname{Hom}_{A^{\prime} \otimes A}\left(A^{\prime} \otimes A, A^{\prime} \otimes A\right)$ via the isomorphism

$$
\operatorname{Hom}(A, A) \cong \operatorname{Hom}_{A^{\prime} \otimes A}\left(A^{\prime} \otimes A, A^{\prime} \otimes A\right)
$$

in (3-9). It therefore suffices to check that $g: A \rightarrow A$ is a morphism of algebras if and only if $\delta_{3}^{A}(g)=g \otimes g$.

From (2-4), we know that

$$
\operatorname{Hom}(A, A) \cong \operatorname{Hom}_{A}(A \otimes A, A) .
$$

Further, from Lemma 4.3, we know that the isomorphism in (4-22) restricts to an isomorphism

$$
\operatorname{Hom}_{\mathrm{Alg}}(A, A) \cong \operatorname{Hom}_{A-A l g}(A \otimes A, A)
$$

From the proof of Proposition 3.5, we also know that the comultiplication $\delta_{3}^{A}$ on $\operatorname{Hom}(A, A)$ is induced by the comultiplication on $\operatorname{Hom}_{A}(A \otimes A, A)$, also denoted $\delta_{3}^{A}$. Hence if $g \in \operatorname{Hom}(A, A)$ corresponds to $g^{\prime} \in \operatorname{Hom}_{A}(A \otimes A, A), \delta_{3}^{A}(g)=g \otimes g$ if and only if $\delta_{3}^{A}\left(g^{\prime}\right)=g^{\prime} \otimes g^{\prime}$.

Applying the result of part (a) to the $A$-algebra $A \otimes A$ in $A$-Mod, it follows that $g^{\prime}: A \otimes A \rightarrow A$ is a morphism of $A$-algebras, i.e., $g^{\prime} \in \operatorname{Hom}_{A-A l g}(A \otimes A, A)$ if and only if $\delta_{3}^{A}\left(g^{\prime}\right)=g^{\prime} \otimes g^{\prime}$. Since $\operatorname{Hom}_{\mathrm{Alg}}(A, A) \cong \operatorname{Hom}_{A-A l g}(A \otimes A, A)$, the result follows.

\section{Analogue of Deligne's lemma}

We will now complete the proof of Theorem 1.2 stated in the introduction.

Proposition 5.1. Let $G=\mathfrak{s p e c}(A)$ be an affine commutative group scheme free of finite rank $r$. Let $A^{\prime}=\underline{\operatorname{Hom}}(A, 1)$. Then, the morphism

$$
N_{A^{\prime}}: \operatorname{Hom}_{A^{\prime} \otimes A}\left(A^{\prime} \otimes A, A^{\prime} \otimes A\right) \rightarrow \operatorname{Hom}_{A^{\prime}}\left(A^{\prime}, A^{\prime}\right)
$$

restricts to a homomorphism of groups from $G(A)$ to $G(1)$.

Proof. Let $f \in G(A) \subseteq \operatorname{Hom}(A, A) \cong \operatorname{Hom}_{A^{\prime} \otimes A}\left(A^{\prime} \otimes A, A^{\prime} \otimes A\right)$, i.e., $f$ is a morphism of algebras. From Proposition 4.4, we know that $\delta_{3}^{A}(f)=f \otimes f$. 
We consider the morphism $\Delta_{A^{\prime}}: A^{\prime} \rightarrow A^{\prime} \otimes A^{\prime}$ of algebras in $\mathbf{C}$. It follows from (4-15) that we have a commutative diagram

$\operatorname{Hom}_{A^{\prime} \otimes A}\left(A^{\prime} \otimes A, A^{\prime} \otimes A\right) \stackrel{\operatorname{Hom}_{A}\left(\Delta_{A^{\prime}} \otimes 1\right)}{\longrightarrow} \operatorname{Hom}_{A^{\prime} \otimes A^{\prime} \otimes A}\left(A^{\prime} \otimes A^{\prime} \otimes A, A^{\prime} \otimes A^{\prime} \otimes A\right)$ $(5-1)$

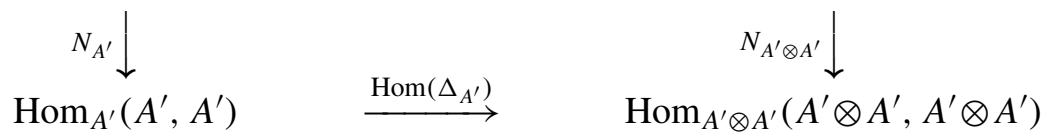

It follows that

$$
\operatorname{Hom}\left(\Delta_{A^{\prime}}\right)\left(N_{A^{\prime}}(f)\right)=N_{A^{\prime} \otimes A^{\prime}}\left(\operatorname{Hom}_{A}\left(\Delta_{A^{\prime}} \otimes 1\right)(f)\right) .
$$

We note that

$$
\Delta_{A^{\prime}} \otimes 1: A^{\prime} \otimes A \rightarrow A^{\prime} \otimes A^{\prime} \otimes A \cong\left(A^{\prime} \otimes A\right) \otimes_{A}\left(A^{\prime} \otimes A\right)
$$

is the coproduct $\Delta^{\prime}:\left(A^{\prime} \otimes A\right) \rightarrow\left(A^{\prime} \otimes A\right) \otimes_{A}\left(A^{\prime} \otimes A\right)$ on the $A$-algebra $A^{\prime} \otimes A$ and hence determines the comultiplication on $\operatorname{Hom}_{A^{\prime} \otimes A}\left(A^{\prime} \otimes A, A^{\prime} \otimes A\right)$. Since $\delta_{3}^{A}(f)=f \otimes f$, it follows from Proposition 4.4 that

(5-4) $\operatorname{Hom}_{A}\left(\Delta_{A^{\prime}} \otimes 1\right)(f)=f \otimes_{A} f:\left(A^{\prime} \otimes A\right) \otimes_{A}\left(A^{\prime} \otimes A\right)=A^{\prime} \otimes A^{\prime} \otimes A$

$$
\longrightarrow A^{\prime} \otimes A^{\prime} \otimes A=\left(A^{\prime} \otimes A\right) \otimes_{A}\left(A^{\prime} \otimes A\right) .
$$

The morphism $f \otimes_{A} f$ in (5-4) can be described by the composition

$$
\left(A^{\prime} \otimes A\right) \otimes_{A}\left(A^{\prime} \otimes A\right) \stackrel{f \otimes_{A} 1}{\longrightarrow}\left(A^{\prime} \otimes A\right) \otimes_{A}\left(A^{\prime} \otimes A\right) \stackrel{1 \otimes_{A} f}{\longrightarrow}\left(A^{\prime} \otimes A\right) \otimes_{A}\left(A^{\prime} \otimes A\right) .
$$

Consider the morphism $e_{A^{\prime}}^{\prime}: A^{\prime} \rightarrow A^{\prime} \otimes A^{\prime}$ of algebras obtained by base changing the unit morphism $e_{A^{\prime}}: 1 \rightarrow A^{\prime}$ with $A^{\prime}$. Then, we have a commutative diagram

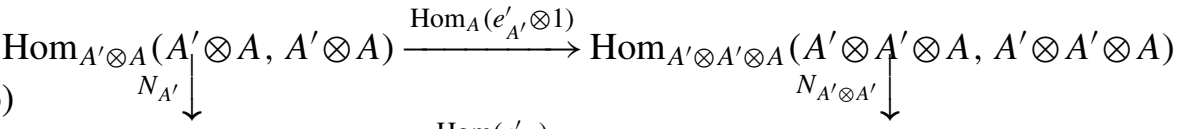

$$
\begin{aligned}
& \operatorname{Hom}_{A^{\prime}}\left(A^{\prime}, A^{\prime}\right) \quad \stackrel{\operatorname{Hom}\left(e_{A^{\prime}}^{\prime}\right)}{\longrightarrow} \quad \operatorname{Hom}_{A^{\prime} \otimes A^{\prime}}\left(A^{\prime} \otimes A^{\prime}, A^{\prime} \otimes A^{\prime}\right)
\end{aligned}
$$

From (5-6), it follows that

$$
1 \otimes N_{A^{\prime}}(f)=N_{A^{\prime} \otimes A^{\prime}}(1 \otimes f)
$$

Now, the comultiplication on $\operatorname{Hom}_{A^{\prime}}\left(A^{\prime}, A^{\prime}\right)$ is induced by the morphism $\operatorname{Hom}\left(\Delta_{A^{\prime}}\right)$ in (5-1). We also note that $1 \otimes_{A} f:\left(A^{\prime} \otimes A\right) \otimes_{A}\left(A^{\prime} \otimes A\right) \rightarrow\left(A^{\prime} \otimes A\right) \otimes_{A}\left(A^{\prime} \otimes A\right)$ is identical to $1 \otimes f: A^{\prime} \otimes A^{\prime} \otimes A \rightarrow A^{\prime} \otimes A^{\prime} \otimes A$. Hence, we have

$$
\begin{aligned}
\delta_{1}\left(N_{A^{\prime}}(f)\right) & =\operatorname{Hom}\left(\Delta_{A^{\prime}}\right)\left(N_{A^{\prime}}(f)\right)=N_{A^{\prime} \otimes A^{\prime}}\left(\operatorname{Hom}_{A}\left(\Delta_{A^{\prime}} \otimes 1\right)(f)\right) \\
& =N_{A^{\prime} \otimes A^{\prime}}\left(f \otimes_{A} f\right)=N_{A^{\prime} \otimes A^{\prime}}\left(f \otimes_{A} 1\right) N_{A^{\prime} \otimes A^{\prime}}\left(1 \otimes_{A} f\right) \\
& =N_{A^{\prime} \otimes A^{\prime}}(f \otimes 1) N_{A^{\prime} \otimes A^{\prime}}(1 \otimes f)=\left(N_{A^{\prime}}(f) \otimes 1\right)\left(1 \otimes N_{A^{\prime}}(f)\right) \\
& =N_{A^{\prime}}(f) \otimes N_{A^{\prime}}(f),
\end{aligned}
$$


and it now follows from Proposition 4.4(a) that $N_{A^{\prime}}(f): A^{\prime} \rightarrow A^{\prime}$ corresponds to a morphism of algebras from $A$ to 1 under the isomorphism

$$
\operatorname{Hom}(A, 1) \cong \operatorname{Hom}_{A^{\prime}}\left(A^{\prime}, A^{\prime}\right) .
$$

Hence, given $f \in G(A)$, it follows that $N_{A^{\prime}}(f) \in G(1)$. It is also clear that $N_{A^{\prime}}$ : $G(A) \rightarrow G(1)$ is a homomorphism.

The result of Proposition 5.1 can be restated as follows: the morphism $N_{A^{\prime}}$ : $\operatorname{Hom}_{A^{\prime} \otimes A}\left(A^{\prime} \otimes A, A^{\prime} \otimes A\right) \rightarrow \operatorname{Hom}_{A^{\prime}}\left(A^{\prime}, A^{\prime}\right)$ restricts to a homomorphism $N$ : $G(A) \rightarrow G(1)$ that fits into a commutative diagram

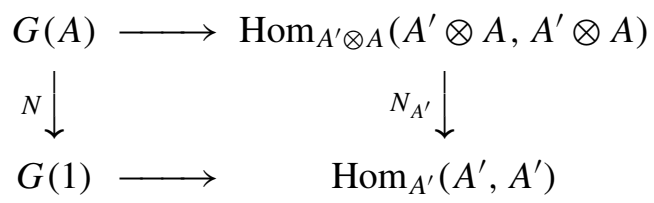

We choose any $u \in G(1)$, i.e. a morphism $u: A \rightarrow 1$ of algebras. For any algebra $B$, the unit map $e_{B}: 1 \rightarrow B$ induces a morphism $e_{B *}: G(1) \rightarrow G(B)$ and hence we can consider the translation map

$$
t_{u, B}: G(B) \rightarrow G(B)
$$

obtained by multiplication with the element $e_{B *}(u)$. By Yoneda lemma, the translations $t_{u, B}$ determine an automorphism $e_{*}(u): A \rightarrow A$ of algebras. We denote the $A^{\prime}$-linear automorphism $1 \otimes e_{*}(u): A^{\prime} \otimes A \rightarrow A^{\prime} \otimes A$ by $\tau$. Since

$$
u \in \operatorname{Hom}_{\mathrm{Alg}}(A, 1) \subseteq \operatorname{Hom}(A, 1) \cong \operatorname{Hom}_{A^{\prime}}\left(A^{\prime}, A^{\prime}\right),
$$

we will often write $u$ as a morphism $u: A^{\prime} \rightarrow A^{\prime}$ of $A^{\prime}$-modules.

Lemma 5.2. Let $u: A \rightarrow 1$ be a morphism of algebras and let

$$
\tau:=1 \otimes e_{*}(u): A^{\prime} \otimes A \rightarrow A^{\prime} \otimes A
$$

be as described above. Then, $N_{A^{\prime}}(\tau)=u^{r}$ where $u^{r}$ denotes the $r$-th power of $u$ as an element of the group $G(1)$.

Proof. We know that $\tau: A^{\prime} \otimes A \rightarrow A^{\prime} \otimes A$ is induced by $u \in \operatorname{Hom}_{\mathrm{Alg}}(A, 1)$ and that $A^{\prime} \otimes A \cong A^{\prime \oplus r}$ in $A^{\prime}$-Mod. From the proof of Lemma 4.1, we know that $N_{A^{\prime}}(\tau) \in \operatorname{Hom}_{A^{\prime}}\left(A^{\prime}, A^{\prime}\right)$ corresponds to the morphism

$$
N_{A^{\prime}}(\tau): \bigwedge_{A^{\prime}}^{r} A^{\prime \oplus r} \cong A^{\prime} \rightarrow \bigwedge_{A^{\prime}}^{r} A^{\prime \oplus r} \cong A^{\prime} .
$$

On each individual summand in $A^{\prime \oplus r}$, the action of the morphism $\tau: A^{\prime \oplus r} \rightarrow A^{\prime \oplus r}$ is induced by $u: A^{\prime} \rightarrow A^{\prime}$. Hence, it follows from (4-8) in the proof of Lemma 4.1 
that the induced action of $\tau$ on the exterior product $\bigwedge_{A^{\prime}}^{r} A^{\prime \oplus r}$ is given by

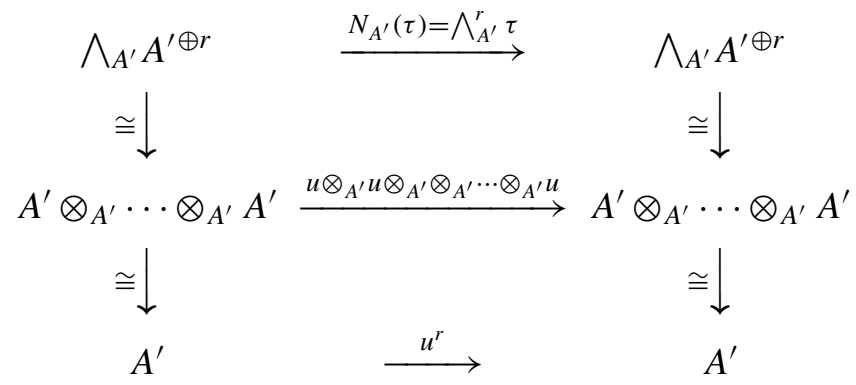

The morphism $u^{r} \in \operatorname{Hom}_{A^{\prime}}\left(A^{\prime}, A^{\prime}\right)$ in (5-12) corresponds to the $r$-th power of

$$
u \in \operatorname{Hom}_{\mathrm{Alg}}(A, 1) \subseteq \operatorname{Hom}(A, 1) \cong \operatorname{Hom}_{A^{\prime}}\left(A^{\prime}, A^{\prime}\right)
$$

as an element of $G(1)=\operatorname{Hom}_{\mathrm{Alg}}(A, 1)$.

Proposition 5.3. Let $G=\mathfrak{s p e c}(A)$ be an affine commutative group scheme free of finite rank $r$. Then, every element of the group $G(1)$ can be annihilated by raising to the $r$-th power.

Proof. We choose any $u \in G(1)$ and let $\tau: A^{\prime} \otimes A \rightarrow A^{\prime} \otimes A$ be as above.

Now, suppose that we have a morphism $f \in \operatorname{Hom}_{A^{\prime} \otimes A}\left(A^{\prime} \otimes A, A^{\prime} \otimes A\right)$. We set $f^{\prime}$ to be the composition

$$
1 \stackrel{e_{A^{\prime} \otimes A}}{\longrightarrow} A^{\prime} \otimes A \stackrel{f}{\longrightarrow} A^{\prime} \otimes A .
$$

Then, from the proof of Proposition 3.1, we know that $f$ is equal to the composition

$$
A^{\prime} \otimes A \cong A^{\prime} \otimes A \otimes 1 \stackrel{1 \otimes f^{\prime}}{\longrightarrow} A^{\prime} \otimes A \otimes A^{\prime} \otimes A \stackrel{m_{A} \otimes m_{A^{\prime}}}{\longrightarrow} A^{\prime} \otimes A .
$$

We set $f_{\tau}:=\tau \circ f: A^{\prime} \otimes A \rightarrow A^{\prime} \otimes A$ and denote by $f_{\tau}^{\prime}$ the composition $A^{\prime} \otimes A \otimes 1 \stackrel{1 \otimes e_{A^{\prime} \otimes A}}{\longrightarrow} A^{\prime} \otimes A \otimes A^{\prime} \otimes A \stackrel{1 \otimes f_{\tau}}{\longrightarrow} A^{\prime} \otimes A \otimes A^{\prime} \otimes A \stackrel{m_{A} \otimes m_{A^{\prime}}}{\longrightarrow} A^{\prime} \otimes A$. It follows that $f_{\tau}^{\prime} \in \operatorname{Hom}_{A^{\prime} \otimes A}\left(A^{\prime} \otimes A, A^{\prime} \otimes A\right)$. We now consider the following commutative diagram in $A^{\prime}$-Mod:
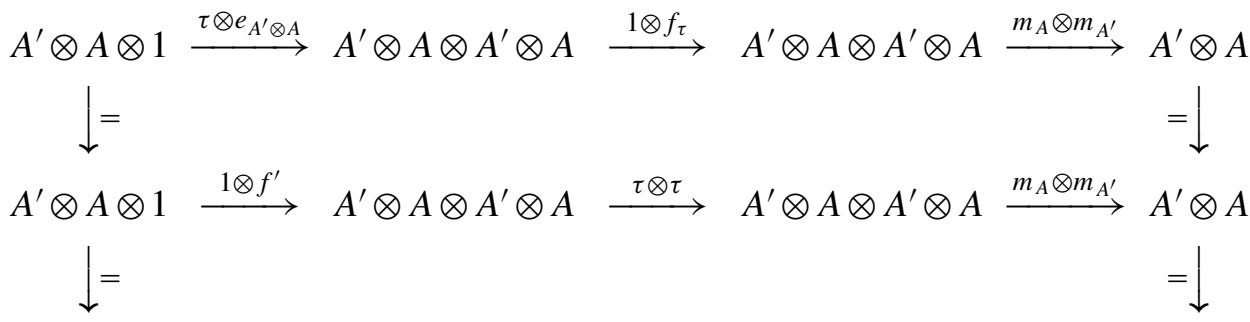

$A^{\prime} \otimes A \otimes 1 \stackrel{1 \otimes f^{\prime}}{\longrightarrow} \quad A^{\prime} \otimes A \otimes A^{\prime} \otimes A \stackrel{m_{A} \otimes m_{A^{\prime}}}{\longrightarrow} \quad A^{\prime} \otimes A \quad \stackrel{\tau}{\longrightarrow} \quad A^{\prime} \otimes A$ 
The upper rectangle in the figure above is commutative because $f_{\tau}=\tau \circ f$, while the lower rectangle commutes because $\tau$ is a morphism of algebras. Identifying $A^{\prime} \otimes A$ with $A^{\prime} \otimes A \otimes 1$, it now follows that

$$
f_{\tau}^{\prime} \circ \tau=\tau \circ f \in \operatorname{Hom}_{A^{\prime}}\left(A^{\prime} \otimes A, A^{\prime} \otimes A\right) .
$$

Since $\tau$ is an automorphism, we have $f_{\tau}^{\prime}=\tau f \tau^{-1}$. Then, since $\operatorname{Hom}_{A^{\prime}}\left(A^{\prime}, A^{\prime}\right)$ is commutative,

$$
N_{A^{\prime}}\left(f_{\tau}^{\prime}\right)=N_{A^{\prime}}\left(\tau f \tau^{-1}\right)=N_{A^{\prime}}(\tau f) N_{A^{\prime}}\left(\tau^{-1}\right)=N_{A^{\prime}}\left(\tau^{-1}\right) N_{A^{\prime}}(\tau f)=N_{A^{\prime}}(f) .
$$

We also note that if $h_{1}, h_{2} \in G(A)=\operatorname{Hom}_{\mathrm{Alg}}(A, A)$ are two morphisms of algebras, the product $h_{1} * h_{2} \in G(A)$ corresponds to the morphism

$$
h_{1} * h_{2}: A \stackrel{\Delta_{A}}{\longrightarrow} A \otimes A \stackrel{h_{1} \otimes h_{2}}{\longrightarrow} A \otimes A \stackrel{m_{A}}{\longrightarrow} A .
$$

We have an isomorphism

$$
H: \operatorname{Hom}(A, A) \stackrel{\cong}{\longrightarrow} \operatorname{Hom}_{A^{\prime} \otimes A}\left(A^{\prime} \otimes A, A^{\prime} \otimes A\right) .
$$

In particular, let $f=H\left(1_{A}\right)$. Then, we have $f_{\tau}^{\prime}=H\left(\left(1_{A} \otimes u\right) \circ \Delta_{A}\right)$. Now, since $N_{A^{\prime}}(f)=N_{A^{\prime}}\left(f_{\tau}^{\prime}\right)$, it follows that

$$
\begin{aligned}
N_{A^{\prime}}(f) & =N_{A^{\prime}}\left(H\left(\left(1_{A} \otimes u\right) \circ \Delta_{A}\right)\right) \\
& =N_{A^{\prime}}\left(H\left(m_{A} \circ\left(1_{A} \otimes e_{*}(u)\right) \circ \Delta_{A}\right)\right) \\
& =N\left(1_{A} * e_{*}(u)\right) \\
& =N\left(1_{A}\right) * N\left(e_{*}(u)\right)=N_{A^{\prime}}(f) * N_{A^{\prime}}(\tau),
\end{aligned}
$$

where the products $N\left(1_{A}\right) * N\left(e_{*}(u)\right)$ and $N_{A^{\prime}}(f) * N_{A^{\prime}}(\tau)$ are taken in $G(1)$. Finally, from Lemma 5.2, we know that $N_{A^{\prime}}(\tau)=u^{r} \in G(1)$. Combining with (5-17), it follows that $u^{r}$ is the identity element of the group $G(1)$.

Theorem 5.4. Let $G=\operatorname{spec}(A)$ be an affine commutative group scheme free of finite rank $r$. Then, for any algebra $B$ in $\mathbf{C}$ and any element $u \in G(B)$, we have $u^{r}=1_{B}$, where $1_{B}$ denotes the identity element of $G(B)$.

Proof. For any algebra $B$ in $\mathbf{C}$, we consider the symmetric monoidal category $\left(B-M o d, \otimes_{B}, B\right)$. Then, if we set $B_{A}:=B \otimes A$, the functor $\operatorname{Hom}_{B-A l g}\left(B_{A},-\right)$ defines an affine commutative group scheme $G_{B}$ on $B$-Mod free of finite rank $r$.

From Proposition 5.3, it now follows that all elements in the group $G_{B}(B)$ are annihilated by raising to the $r$-th power. Further, from Lemma 4.3, it follows that $G_{B}(B)=\operatorname{Hom}_{B-A l g}(B \otimes A, B) \cong \operatorname{Hom}_{\mathrm{Alg}}(A, B)=G(B)$. This proves the result. 


\section{References}

[Deligne 1990] P. Deligne, "Catégories tannakiennes", pp. 111-195 in The Grothendieck Festschrift, vol. 2, edited by P. Cartier et al., Progr. Math. 87, Birkhäuser, Boston, MA, 1990. MR 92d:14002 Zbl 0727.14010

[Demazure and Gabriel 1970] M. Demazure and P. Gabriel, Groupes algébriques, I: géométrie algébrique, généralités, groupes commutatifs, Masson, Paris, 1970. MR 46 \#1800 Zbl 0203.23401

[Hakim 1972] M. Hakim, Topos annelés et schémas relatifs, Ergebnisse der Math. 64, Springer, Berlin, 1972. MR 51 \#500 Zbl 0246.14004

[Mac Lane 1998] S. Mac Lane, Categories for the working mathematician, 2nd ed., Graduate Texts in Mathematics 5, Springer, New York, 1998. MR 2001j:18001 Zbl 0906.18001

[May 2001] J. P. May, "Picard groups, Grothendieck rings, and Burnside rings of categories", $A d v$. Math. 1 (2001), 1-16. MR 2002k:18011 Zbl 0994.18004

[Tate 1997] J. Tate, "Finite flat group schemes", pp. 121-154 in Modular forms and Fermat's last theorem (Boston, 1995), edited by G. Cornell et al., Springer, New York, 1997. MR 1638478 Zbl 0924.14024

[Tate and Oort 1970] J. Tate and F. Oort, "Group schemes of prime order", Ann. Sci. École Norm. Sup. (4) 3 (1970), 1-21. MR 42 \#278 Zbl 0195.50801

[Toën and Vaquié 2009] B. Toën and M. Vaquié, "Au-dessous de Spec ZZ", J. K-Theory 3:3 (2009), 437-500. MR 2010j:14006 Zbl 1177.14022

[Waterhouse 1979] W. C. Waterhouse, Introduction to affine group schemes, Graduate Texts in Mathematics 66, Springer, New York, 1979. MR 82e:14003 Zbl 0442.14017

Received May 29, 2011. Revised September 25, 2011.

ABHISHEK BANERJEE

DEPARTMENT OF MATHEMATICS

OHIO STATE UNIVERSITY

$231 \mathrm{~W} 18 \mathrm{TH}$ AVENUE

COLumbus, OH 43210

UNITED STATES

abhishekbanerjee1313@gmail.com 


\title{
PACIFIC JOURNAL OF MATHEMATICS
}

\author{
http://pacificmath.org \\ Founded in 1951 by \\ E. F. Beckenbach (1906-1982) and F. Wolf (1904-1989)
}

\section{EDITORS}

V. S. Varadarajan (Managing Editor)

Department of Mathematics

University of California

Los Angeles, CA 90095-1555

pacific@math.ucla.edu

Vyjayanthi Chari

Department of Mathematics

University of California

Riverside, CA 92521-0135

chari@math.ucr.edu

\section{Robert Finn}

Department of Mathematics Stanford University

Stanford, CA 94305-2125

finn@math.stanford.edu

Kefeng Liu

Department of Mathematics

University of California

Los Angeles, CA 90095-1555

liu@math.ucla.edu
Darren Long

Department of Mathematics

University of California

Santa Barbara, CA 93106-3080

long@math.ucsb.edu

Jiang-Hua Lu

Department of Mathematics

The University of Hong Kong

Pokfulam Rd., Hong Kong jhlu@maths.hku.hk

Alexander Merkurjev

Department of Mathematics

University of California

Los Angeles, CA 90095-1555

merkurev@math.ucla.edu
Sorin Popa

Department of Mathematics University of California

Los Angeles, CA 90095-1555 popa@math.ucla.edu

Jie Qing

Department of Mathematics

University of California

Santa Cruz, CA 95064

qing@cats.ucsc.edu

Jonathan Rogawski

Department of Mathematics

University of California

Los Angeles, CA 90095-1555

jonr@math.ucla.edu

\section{PRODUCTION}

pacific@math.berkeley.edu

\section{SUPPORTING INSTITUTIONS}

ACADEMIA SINICA, TAIPEI

CALIFORNIA INST. OF TECHNOLOGY INST. DE MATEMÁTICA PURA E APLICADA KEIO UNIVERSITY

MATH. SCIENCES RESEARCH INSTITUTE NEW MEXICO STATE UNIV.

OREGON STATE UNIV.

\author{
STANFORD UNIVERSITY \\ UNIV. OF BRITISH COLUMBIA \\ UNIV. OF CALIFORNIA, BERKELEY \\ UNIV. OF CALIFORNIA, DAVIS \\ UNIV. OF CALIFORNIA, LOS ANGELES \\ UNIV. OF CALIFORNIA, RIVERSIDE \\ UNIV. OF CALIFORNIA, SAN DIEGO \\ UNIV. OF CALIF., SANTA BARBARA
}

\author{
UNIV. OF CALIF., SANTA CRUZ \\ UNIV. OF MONTANA \\ UNIV. OF OREGON \\ UNIV. OF SOUTHERN CALIFORNIA \\ UNIV. OF UTAH \\ UNIV. OF WASHINGTON \\ WASHINGTON STATE UNIVERSITY
}

These supporting institutions contribute to the cost of publication of this Journal, but they are not owners or publishers and have no responsibility for its contents or policies.

See inside back cover or pacificmath.org for submission instructions.

The subscription price for 2012 is US \$420/year for the electronic version, and \$485/year for print and electronic.

Subscriptions, requests for back issues from the last three years and changes of subscribers address should be sent to Pacific Journal of Mathematics, P.O. Box 4163, Berkeley, CA 94704-0163, U.S.A. Prior back issues are obtainable from Periodicals Service Company, 11 Main Street, Germantown, NY 12526-5635. The Pacific Journal of Mathematics is indexed by Mathematical Reviews, Zentralblatt MATH, PASCAL CNRS Index, Referativnyi Zhurnal, Current Mathematical Publications and the Science Citation Index.

The Pacific Journal of Mathematics (ISSN 0030-8730) at the University of California, c/o Department of Mathematics, 969 Evans Hall, Berkeley, CA 94720-3840, is published monthly except July and August. Periodical rate postage paid at Berkeley, CA 94704, and additional mailing offices. POSTMASTER: send address changes to Pacific Journal of Mathematics, P.O. Box 4163, Berkeley, CA 94704-0163.

PJM peer review and production are managed by EditFLOW ${ }^{\mathrm{TM}}$ from Mathematical Sciences Publishers.

PUBLISHED BY PACIFIC JOURNAL OF MATHEMATICS

at the University of California, Berkeley 94720-3840

A NON-PROFIT CORPORATION

Typeset in LATEX

Copyright $(02012$ by Pacific Journal of Mathematics 


\section{PACIFIC JOURNAL OF MATHEMATICS}

Volume $255 \quad$ No. $1 \quad$ January 2012

Averaging sequences

FERnANDo Alcalde Cuesta and Ana Rechtman

Affine group schemes over symmetric monoidal categories

ABHISHEK BANERJEE

Eigenvalue estimates on domains in complete noncompact Riemannian

manifolds

Daguang Chen, TAO Zheng and Min LU

Realizing the local Weil representation over a number field

GERALD CLIFF and DAVID MCNEILLY

Lagrangian submanifolds in complex projective space with parallel second fundamental form

Franki DiLlen, Haizhong Li, LuC VRANCKEN and XiANFENG

WANG

Ultra-discretization of the $D_{4}^{(3)}$-geometric crystal to the $G_{2}^{(1)}$-perfect crystals

Mana Igarashi, KaIlash C. MisRa and ToshiKi NAKASHIMA Connectivity properties for actions on locally finite trees

KEITH JONES

Remarks on the curvature behavior at the first singular time of the Ricci flow

NAM Q. LE and NATASA SESUm

Stability of capillary surfaces with planar boundary in the absence of gravity

PetKo I. MARINOV

Small hyperbolic polyhedra

SHAWN RAFALSKI

Hurwitz spaces of coverings with two special fibers and monodromy group 241 a Weyl group of type $B_{d}$

FRANCESCA VETRO 